\title{
Visual realism enhances realistic response in an immersive virtual environment - Part 2
}

\author{
Insu $\mathrm{Yu}^{1}$, Jesper Mortensen ${ }^{1,2}$, Pankaj Khanna ${ }^{1}$, Mel Slater*1,3 \\ ${ }^{1}$ Department of Computer Science, University College London, UK \\ ${ }^{2}$ Geomerics, Cambridge, UK \\ ${ }^{3}$ ICREA-University of Barcelona, Faculty of Psychology, Barcelona, Spain. \\ *Corresponding author: Mel Slater, melslater@ub.edu
}

\begin{abstract}
Does realistic lighting in an immersive virtual reality application enhance presence, where participants feel that they are in the scene and behave correspondingly? Our previous study indicated that presence is more likely with real-time ray tracing compared with ray casting, but we could not separate the effects of overall quality of illumination from the dynamic effects of real-time shadows and reflections. Here we describe an experiment where 20 people experienced a scene rendered with global or local illumination. However, in both conditions there were dynamically changing shadows and reflections. We found that the quality of illumination did not impact presence, so that the earlier result must have been due to dynamic shadows and reflections. However, global illumination resulted in greater plausibility participants were more likely to respond as if the virtual events were real. We conclude that global illumination does impact the responses of participants and is worth the effort.
\end{abstract}

\section{Keywords}

Virtual environments, presence, visual realism, real-time global illumination, shadows, reflections, virtual body, avatar.

\section{Introduction}

In this article we consider the impact of realistic illumination on 'presence' in immersive virtual reality (VR), continuing work first reported Part 1 [1]. In particular we consider whether illumination realism in itself contributes to presence, or whether this is only because realistic lighting also includes dynamic 
effects such as changing shadows and reflections as the participant moves around the scene. First we briefly consider the notion of presence, and then describe the new experiment in detail. Putting together the results of the new experiment with those of Part 1, we arrive at the originally unexpected conclusion that indeed realistic lighting and dynamic effects are both important contributors to presence, though for different reasons.

People experiencing VR may have the illusion of being in the virtual place, and consequently carry out actions as if the situation and events depicted were really happening. These actions may include non-conscious ones, including changes in physiology such as heart rate, behavioral ones such as smiling at a virtual human character, through to how they feel emotionally, and the types of thoughts they have. This sensation of being in the virtual place, operationalized by the response to the virtual environment as if it were real, is referred to as presence, a concept with a long history with roots in teleoperator systems, see [2]. What is of interest to practitioners in the field, and application builders, is to understand how the actual properties of the virtual reality system used may influence the sensation of presence of an average VR participant.

One obvious factor that from a common-sense point of view ought to contribute to presence is how real the depicted scene looks. There are many possible meanings to 'real', but here we concentrate specifically on illumination realism - and consider how much does the realism generated by the illumination model used in the rendering of the scene contribute to presence?

On the face of it the answer would seem to be obvious - if presence is about people responding realistically to a virtual environment scenario, then the more the illumination conforms to reality the more likely it is that they will respond realistically. However, surprisingly, some past data does not support that viewpoint. We have covered this issue in detail in Part 1 of this article [1], but we recall one example. Zimmonds and Panter from UNC Chapel Hill [3] carried out an experiment where participants each experienced one of five different types of illumination rendering, ranging from wire frame to radiosity, in the 'pit room' environment. The pit room is where participants enter a virtual room that has a precipice cut out in its center, with a significant drop to another floor below. (We gave a brief history of the pit room environment in Part 1 of this article). Measures of presence were by questionnaire and also heart rate changes in response to the possible stress caused by the precipice. It was found that there were no differences in subjective score or in physiological response to the different illumination types: wire frame rendering produced the same result as radiosity. However, under all illumination types participants had an increase in heart rate when they encountered the pit. There was presence, but it was not related to the type of illumination used.

In Part 1 of this article we described an experiment that also utilized a version of the pit room [1]. In that experiment we compared presence in a scene illuminated by a ray casting method of illumination with the same scene illuminated by real-time ray 
tracing. In ray casting only one ray is traced from the viewpoint into the scene for every pixel (or sub-pixel) and the illumination is determined solely by the direct light effect on the first surface intersected by the ray. In ray tracing the first ray through the pixel follows a subsequent path determined by specular surfaces, so that it is reflected from mirror surfaces, and includes shadow rays. Hence ray casting results in local illumination effects only, with no shadows, whereas ray tracing results in a portrayal of light inter-reflection between specularly reflecting surfaces and also of highlights and reflections that dynamically change according to the head position and gaze direction of the participant. The participants perceived the scene through a head-tracked stereo head-mounted display and they were endowed with a simple virtual body. Since the ray tracing was executed in real time their virtual body therefore cast shadows and reflections in the ray tracing case that moved as they moved. This meant, for example, that as they looked down into the pit they would see a shadow of their virtual body moving as they moved. The fundamental distinction, therefore, between the ray casting and ray tracing methods was that the latter produced dynamic illumination effects (real-time moving shadows and mirror reflections) and the former had no shadows or reflections at all.

In that experiment presence was assessed through questionnaire and physiological responses to being in the pit room. It was found that both presence questionnaires and physiological responses indicating anxiety were higher for those who experienced the ray tracing condition. Hence, in terms of presence, the ray tracing method apparently produced the better effect.

We suspected that this result was due to the dynamic nature of the illumination in the ray tracing condition - especially the fact that the moving shadows and reflections were caused by and correlated with head and body movements of the participants. However, we could not rule out the possibility that it was the overall higher illumination quality of the ray-traced environment that led to the significantly higher presence in the ray tracing case. Remember that the ray tracing not only had dynamically changing reflections and shadows, but just from the static point of view the illumination would appear to be more realistic.

In this paper we describe an experiment that was designed to settle this issue - to determine whether the better result for ray tracing was caused by overall improvement in appearance or by the dynamic changes. As before we have compared presence in a virtual room under two conditions but where both had dynamic shadows and reflections. In one condition the room was illuminated using standard OpenGL Gouraud interpolated shading but including dynamically changing shadow umbras and reflections. In the second condition it was rendered with global illumination, that of course had soft shadows and mirror reflections, but also included other global illumination effects such as color bleeding. Since there were dynamic changes in both conditions, any difference in result would be due solely to the overall quality of the illumination. 
What we expected to find is that there would be no difference in presence between these two conditions, since the dynamic aspects of the illumination were the same. This would mean that the overall quality of the illumination is not a critical factor to achieve presence but that dynamic changes to the illumination - at least those caused by the participant's own movements, would be critical. (This assumes that the application itself is not intrinsically bound up with the issue of illumination, as it would be in the case of architectural walkthrough applications).

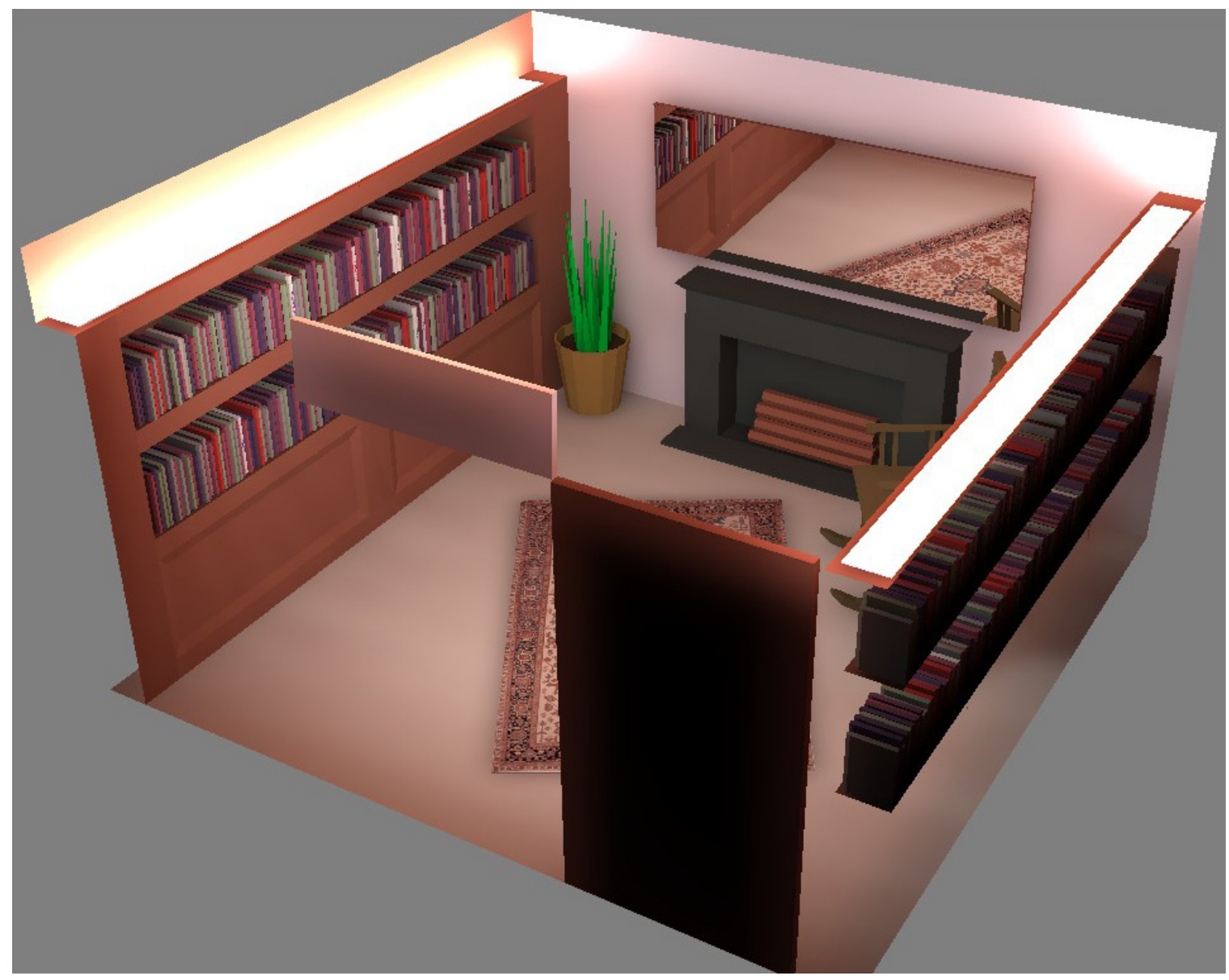

Figure 1 - Overview of the scene. The size of the virtual library was chosen to coincide with the physical size of the Cave system in which it was displayed.

\section{Methods}

\section{The Virtual Reality System}

We implemented and carried out the experiment out using a 5 PC cluster driving a four wall Trimension ReaCToR Cave-like system. (Henceforth, we refer to this using the generic name 'Cave'). Each wall was assigned a dedicated PC for rendering and a 
single master PC was collecting data and running the simulation. The Trimension Reactor system has three $3 \mathrm{~m} \times 2.2 \mathrm{~m}$ back-projected screens: front, left, and right, and a $3 \mathrm{~m} \times 3 \mathrm{~m}$ front projection surface on the floor. The computers in the cluster contained Intel Pentium 3.2 GHz processors with 1 gigabyte of RAM and Nvidia Quadro FX 5600 graphics cards. The participants were fitted with shutter glasses that were synchronized with the projectors delivering active stereo at $45 \mathrm{~Hz}$ each eye. Attached to the top of the glasses was an InterSense IS-900 tracking device to track the head of the participant.

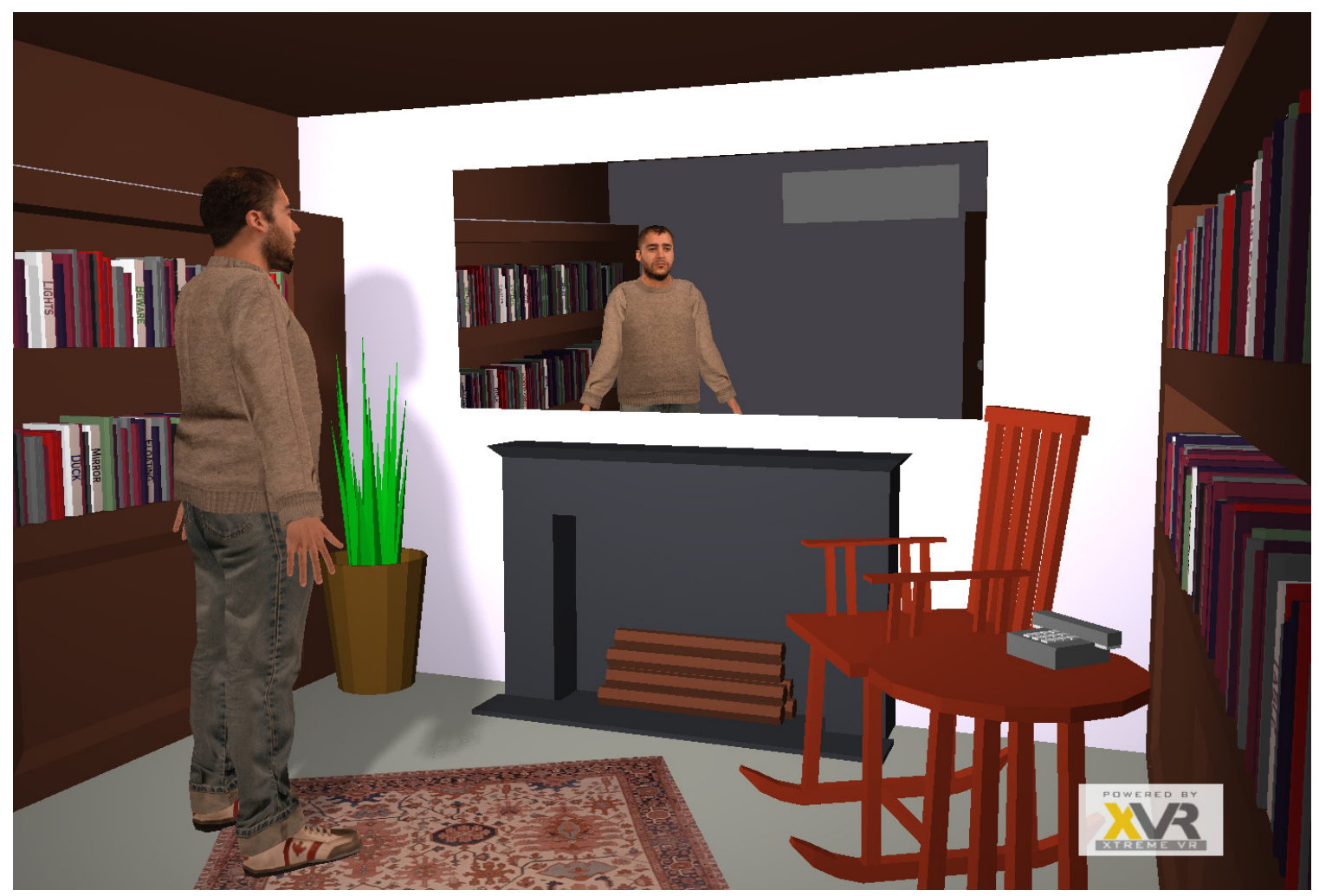

(a) OGL with the male avatar 


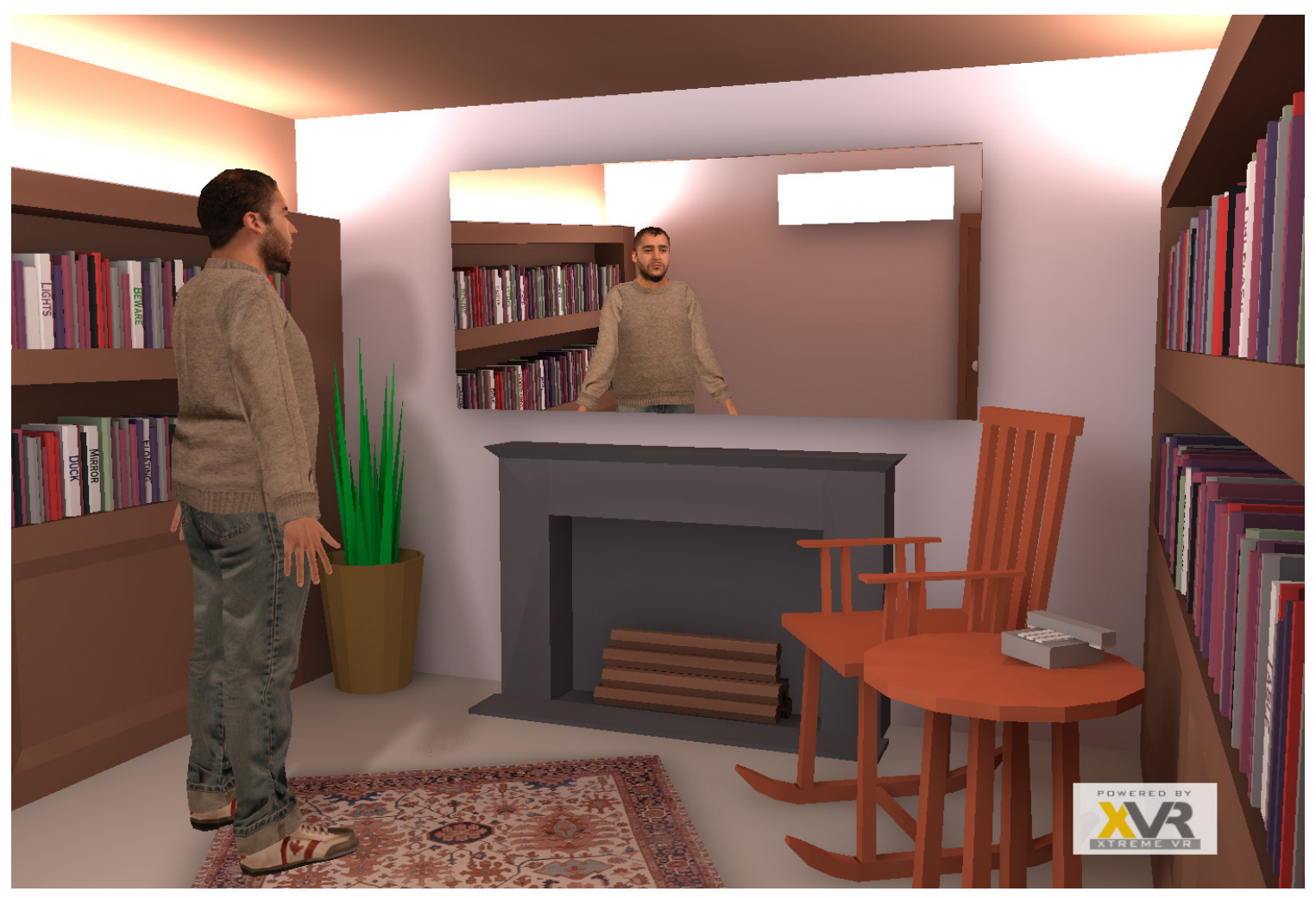

(b) VLF with the male avatar 


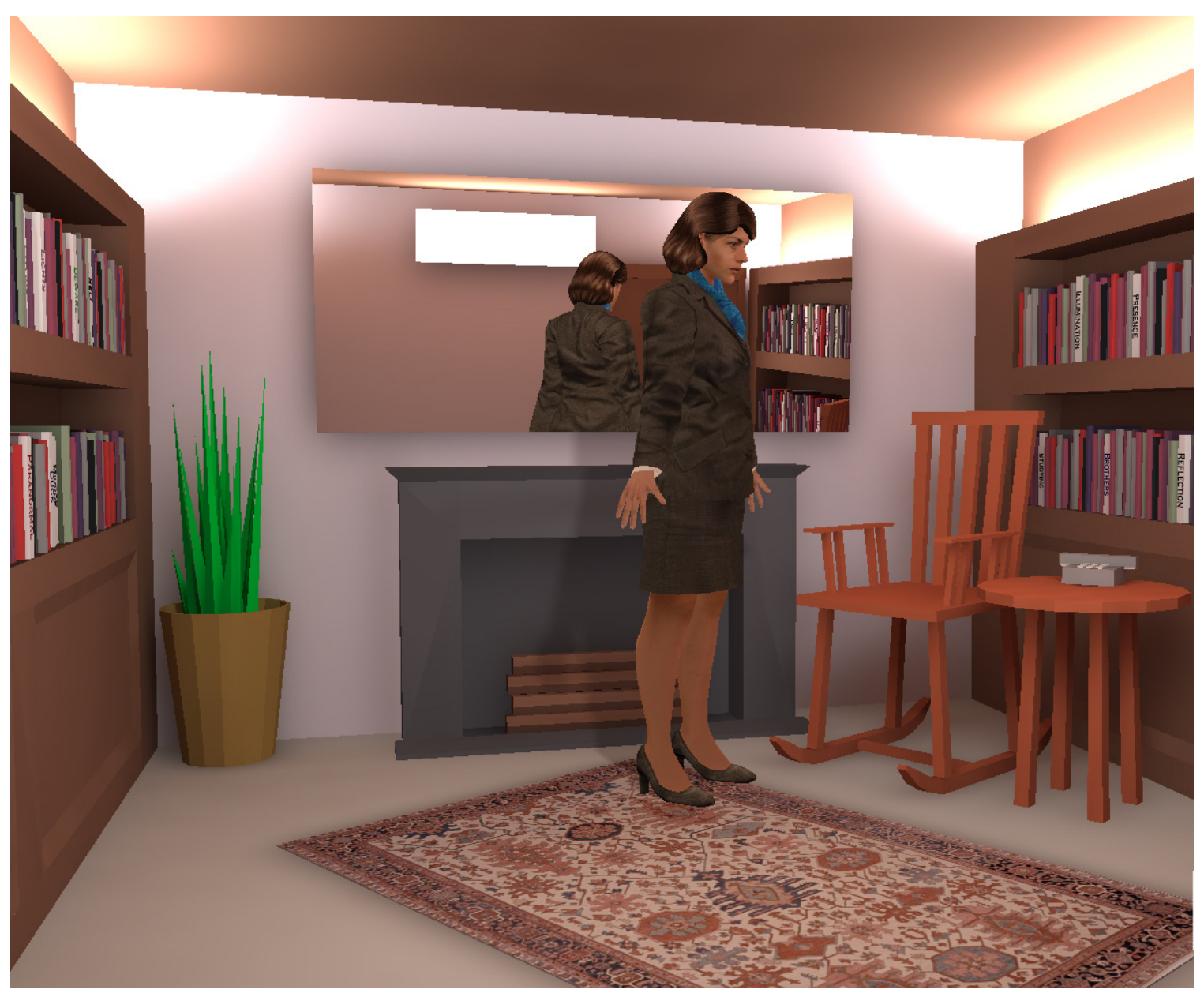

(c) VLF with the female avatar

Figure 2 - The scene rendered with (a) the OGL method and (b) the VLF method showing the male avatar and (c) the female avatar. Dynamic shadows and reflections of the body are shown in all, but (b) and (c) have overall global illumination and (a) only local illumination.

\section{The Scene}

The scene we used for the experiment is shown in Figure 1. The virtual environment depicted a library with furniture, some bookshelves and a large mirror over a fireplace. Three area light sources illuminated the scene. Two were on top of the bookshelves pointing up and another was positioned opposite the mirror on the back wall.

We designed the virtual room to be the same size of the Cave, so that its walls coincided with the Cave walls. The room was oriented so that the mirror was on the 
back wall of the Cave, i.e., the one that participants would naturally face when entering into the Cave.

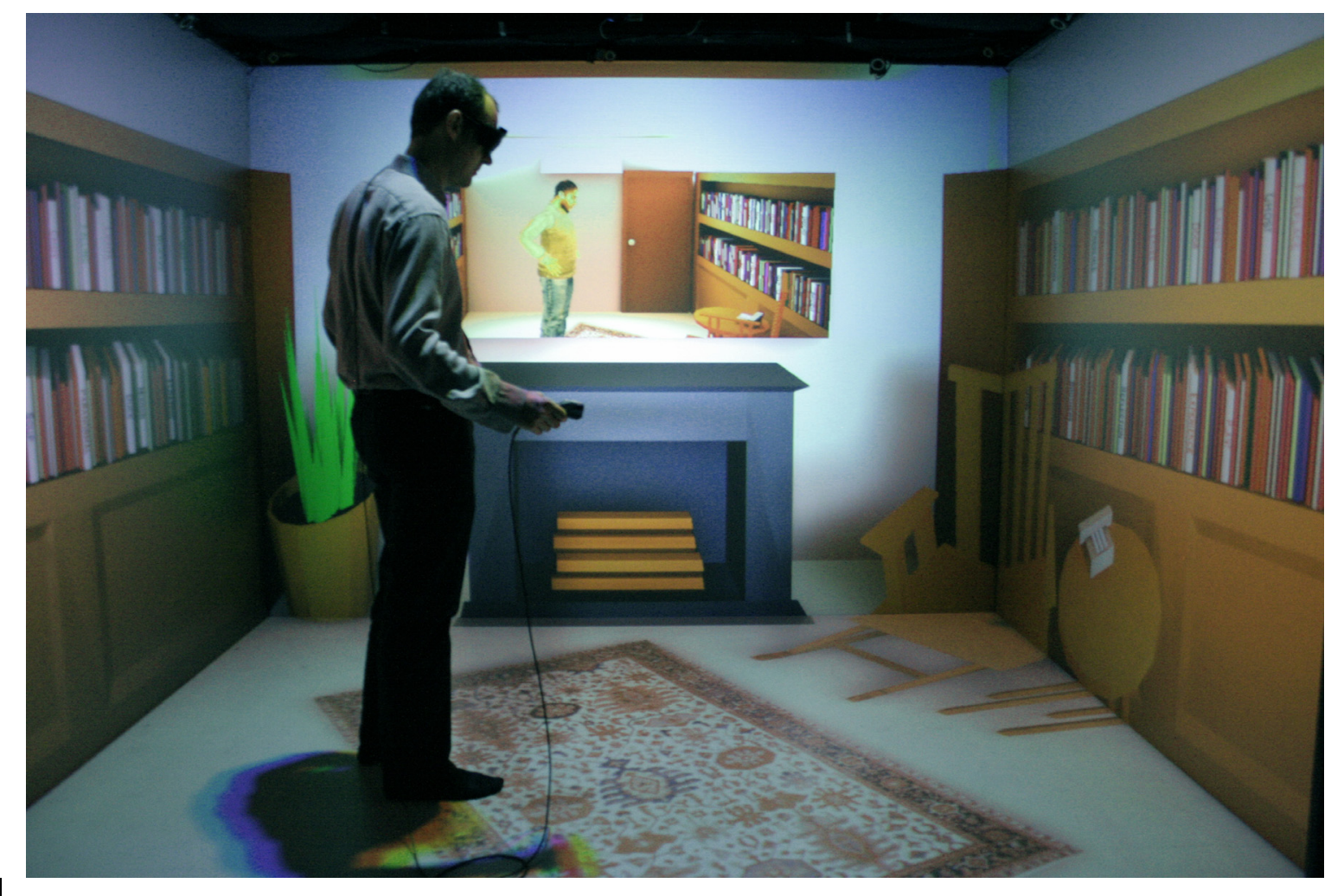

Figure 3 - Photograph of a person in the Cave system, with the room rendered with global illumination. The shadow behind the person is caused by the Cave ceiling projector. A faint virtual shadow of the virtual body of the person can be seen directed towards the wall. A mirror reflection of the avatar representing the person can also be seen.

We used three different rendering methods: The first was simple OpenGL based interpolated shading. This was used for the experimental baseline, and we refer to this as Baseline. The second was the same as the first, but also included dynamic shadows and reflections (Figure 2a). We refer to this as OGL rendering. The third type of rendering was full global illumination using the virtual light field (VLF) method described in the next section (Figure 2b,c). The dynamic shadows and reflections were identical in the OGL and VLF conditions. Figure 3 shows a person in the Cave with the scene rendered using the VLF method. 
The participant was represented by a virtual humanoid character (or avatar). There was a female and a male avatar available depending on the gender of the participant (Figure 2). Since in a Cave participants can see their own real body, the avatar was invisible, except that it could cast shadows and reflections as if it were visible. The avatar was quite detailed comprising approximately $10 \mathrm{~K}$ polygons, textured and hand-rigged for realistic skin deformations, depending on the pose of a skeletal structure embedded within the model. The avatar model was from AXYZ Design (www.axyzdesign.com). It was animated with inverse kinematics derived only from head tracker and a wand, each with 6 degrees of freedom. The IK method also used relative position and angle between the head and the wand to estimate the elbow position. This therefore partially replicated the participant's real body movements, their right hand holding the wand resulted in the avatar's right hand arm moving accordingly, as would be seen in the mirror reflection and shadows of the avatar. Moreover, head-turns were also correctly rendered on the avatar representation, and up and down body moves would also be seen to be reflected in the mirror, as would whole body turns.

\section{The Rendering Method}

In order to provide global illumination at real-time frame rates a we developed a hybrid rendering method. We employed the Virtual Light Field (VLF) [4] in order to account for global illumination effects between the static elements of the scene. This accounts for full $\mathrm{L}\{\mathrm{S}|\mathrm{D}| \mathrm{G}\}^{*} \mathrm{E}$ illumination in static environments, and thus adds color bleeding effects, caustic reflections due to surfaces with non-diffuse BRDFs and soft shadows. The VLF essentially stores a sampling of the outgoing radiance from all points in the scene in all directions and this data can be interpolated to produce renderings from arbitrary vantage points. The VLF for a scene can be computed on a GPU in linear time in the number of polygons and provide solutions with tens of thousands of polygons with millions of (ir)radiance elements in minutes. We could then use the converged VLF for real-time rendering adding only a small constant time cost to a traditional renderer.

Integrating dynamic changes into a global illumination solution is a difficult task. However, by breaking up the problem and attacking the modes of light transport that contribute most to the image we can achieve real-time and still support significant global illumination effects. We can separate the problem into three main modes of transport contributing to the image and focus on solving these: (1) Field radiance scattered off the avatar towards the eye (2) soft shadows cast by the avatar, and (3) specular reflections of the avatar. This does not solve for reflections from the avatar, and thus color bleeding caused by the avatar will not be accounted for. However, the magnitude of illumination that has undergone multiple diffuse reflections is generally low and adds little to the image. 
In order to solve (1) we need to be able to rapidly provide the irradiance at an arbitrary spatial position and direction. In order to provide irradiance calculations at real-time frame rates we use an irradiance volume $[5,6]$. The bounding volume of the scene is subdivided into a set of voxels. A voxel stores irradiance retrieved from the VLF projected to a spherical harmonic (SH). Irradiance values at arbitrary positions can be interpolated from neighboring voxels.

Physically correct soft shadows are notoriously difficult to calculate. However, perceptually correct soft shadows can be rendered in real-time using the graphics processing unit (GPU). We used the Percent Closer Soft Shadows method [7] to sample a standard shadow map stochastically to provide approximate umbra and penumbra regions of a shadow due to an area light source.

Reflections (and caustics) are already appropriately accounted for in the VLF for the static parts of the scene, but this obviously does not include the dynamic elements. This can easily be achieved in real-time using a reflection rendering pass [8], rendering the visible scene and dynamic objects onto a reflective surface which is texture mapped to the specular geometry.

The effect of these techniques used in conjunction is quite striking. The dynamic elements merge well with the surrounding scene featuring impinging color bleeding, caustics and casting soft shadows as well as being visible in reflective surfaces (Figures 2 and 3).

\section{Experimental Design}

The experiment was a within-groups design with two groups. Both groups experienced first the Baseline condition. One group experienced next the OGL condition and subsequently the VLF. The other group experienced their second and third exposures as first VLF and then OGL. This is summarized in Table 1.

We used a within-groups design because we definitely wanted to compare the results of each participant against him- or herself across the two conditions. Unlike the experiment described in Part 1 (which also discusses issues surrounding between- and within-group designs) we believed that the differences between the VLF and OGL conditions were subtle enough that participants would not obviously realize the purpose of the experiment, and therefore their responses would be unlikely to be biased by this knowledge. On the other hand, their responses to the events in the environment could change in their subsequent exposure. However, if we consider only the results of their first exposure after the Baseline (which of course was either OGL or VLF) then this can also be analyzed as a between-groups experiment. 


\section{Table 1 - The Experimental Conditions}

\begin{tabular}{|l|l|l|l|}
\hline Condition & $\begin{array}{l}\text { Illumination } \\
\text { Model }\end{array}$ & $\begin{array}{l}\text { Dynamic Shadows } \\
\text { and Reflections }\end{array}$ & $\begin{array}{l}\text { Actions - books falling } \\
\text { and character appears }\end{array}$ \\
\hline Baseline & $\begin{array}{l}\text { Interpolated } \\
\text { Shading }\end{array}$ & No & No \\
\hline OGL & $\begin{array}{l}\text { Interpolated } \\
\text { Shading }\end{array}$ & Yes & Yes \\
\hline VLF & $\begin{array}{l}\text { Global } \\
\text { illumination }\end{array}$ & Yes & Yes \\
\hline
\end{tabular}

\section{Experimental Procedures}

Twenty one participants were recruited for the experiment ( 6 female), but there were missing data for one which has therefore has been excluded from all analysis presented here. The participants were recruited from around the campus at University College London and they were paid the equivalent of $\$ 10$ for their participation. The experiment was approved by the UCL Ethics Committee under informed consent. We have prepared a video of the main part of the experiment which is available online (www.youtube.com/watch?v=X2A_LVC--N8).

Upon entering each of the environments participants were first invited to look around and report what they saw. This was to ease them into the environment and also for us to check that the equipment was properly working. There was soft music playing in the background.

They first experienced the Baseline condition, in order for them to become adapted to the environment. This Baseline condition simply showed the virtual library, but not the avatar reflections nor any dynamic shadows, and no events occurred. The Baseline lasted for 150s, where the participants simply looked at and moved around the room.

Next they either experienced the VLF condition or the OGL condition according to a pre-determined random order. Their third experience was in the remaining condition. In both their second and third trials the experience was the same, and each lasted for 180s. They saw a reflection in the mirror of an avatar with upper body movements that partially followed their own movements as described above. The task given to the participants was to examine the book titles. They were told that in the book titles there was a clue as to what was happening within the environment. This was only to encourage them to move and look around the environment, and had no other experimental purpose. 
After 1 minute into the second and third trials books started to fall from the bookshelves, controlled by a physics simulation. At the end of the period of falling books, one was left displayed on the head of the mirror avatar reflection. We were curious to see how they would react to this, since an attempt to touch their head to feel the virtual book seen on the mirrored avatar head would be a sign of selfrecognition called the Mirror Test. However, this was not one of the experimental issues. In both second and third exposures after a further 1 minute a virtual character appeared in the room. This character was a boy who floated around the room three times and then disappeared. Participants would typically first see this character in the mirror. The purpose of this event was to inject a shock into the environment, useful for measuring differences in physiological responses between the conditions.

At the end of each of their three experiences the scene was replaced by text containing the questionnaire described in the next section. After answering all of the questions the participants were instructed to close their eyes until the next session started.

Overall the environment had a somewhat strange aspect, with book titles pointing towards paranormal events. Also the books falling and the sudden appearance of the floating boy added to this, as did the background music.

\section{The Presence Questionnaire}

At the end of each session and while the participants were still in the Cave a series of questions was displayed on the Cave walls, and they were asked to score each one on a 0 to 100 scale where 100 was the most positive response. The questions were as follows:

Q1. I sometimes felt myself to be in the library much as if I was in a real place.

Q2. When I think about it now I remember the library more as if it were a place I visited rather than a computer generated world.

Q3. I sometimes forgot about the real world of the laboratory and reacted as if I were in the library.

Q4. I sometimes thought about sitting on the chair.

Q5. I sometimes thought that the plant might be a real one.

Q6. Sometimes my thoughts, feelings and actions were as if I were in a real place.

Q7. Even though the person in the mirror did not look like me I sometimes had the feeling as if I were seeing myself.

Questions Q1, Q2, Q3 and Q6 related to presence. Q4 and Q5 were to examine whether the greater illumination realism would have an impact on the degree of realism of the individual objects. When participants were asked to give their scores during their second and third exposure they were always reminded of their 
previous score for the same question. Q7 was for curiosity to examine overall whether seeing the mirror reflection would generate a feeling of body ownership with respect to the virtual body, an issue that was not a concern of this experiment.

\subsection{Physiological Responses}

The participants were fitted with a Nexus 4 physiological recording device that recorded the ECG. Electrodes were placed on the left and right collar bones and the lowest left rib in order to record ECG.

The ECG in this experiment was acquired as standard Einthoven I derivation (sampling frequency: $1024 \mathrm{~Hz}$ ) and the analysis was performed with the g.BSanalyze biosignal analysis software package (g.tec - Guger Technologies OEG, Graz, Austria). Using this software the QRS (ventricular contraction) complexes were determined, and inspected and manually corrected where necessary. The QRS complexes are the time distances from one heart contraction to the next one. An 'NN interval' means that only normal-to-normal beat distances are retained (non-normal beats such as extra systoles are excluded). The measures retained were:

$\mathrm{HR}$ - the mean heart rate over the 2 minute period (bpm).

NN50 - number of intervals of successive NN intervals greater than $50 \mathrm{~ms}$.

These measured are explained in Insets in [1]. Anxiety is generally exhibited by higher heart rate and lower heart rate variability, here measured by NN50.

Additionally we computed heart rate deceleration (HRD) with respect to $6 \mathrm{~s}$ intervals after the specific event of the boy appearing in the mirror. HRD is the negative of the slope of instantaneous HR measured from just before the onset of the event to the minimum HR attained in the $6 \mathrm{~s}$ after the event. It has been shown to correlate with strong negative feelings [9] (p588).

\section{Results}

\section{Presence Questionnaire Responses}

Figure 4 shows bar charts for the questionnaire responses Q1-Q6 ( $\mathrm{n}=19$, since one subject was removed who gave 0 scores on every question). It should be noted that the scores for the presence related questions Q1-Q3 and Q6 are relatively high overall. It is clear that there is no order effect since the bar charts show the same pattern of responses within both OV and VO. and hence the within-groups design is appropriate. It is also clear that for the questions that relate directly to presence (Q1-Q3 and Q6) there appears to be an increase in score from the Baseline to the experimental conditions, but there is no difference between the experimental 
conditions. The idea of sitting on the chair (Q4) tended to occur to people more during the Baseline but not so much in the subsequent trials. Also the illusion that the plant might be real (Q5) tended to occur more in the VLF condition than in the Baseline or OGL conditions. However, the only strictly valid comparisons are between the OGL and VLF conditions, since the only difference between these was the overall quality of the illumination (in the Baseline there were no events, and no avatar reflection).

Since there are no order effects we can carry out paired tests on the combined data ignoring order in order to compare responses to OGL and VLF (i.e., each participant's scores in one condition is compared with his- or her scores in the other). We use the non-parametric sign test throughout since in the case of the questionnaire responses $n=19$ and the data is ordinal. For the questionnaire responses there are no significant differences on any of the questions between VLF and OGL. The smallest significance levels are $\mathrm{P}=0.11$ for $\mathrm{Q} 5$ and also for Q6.
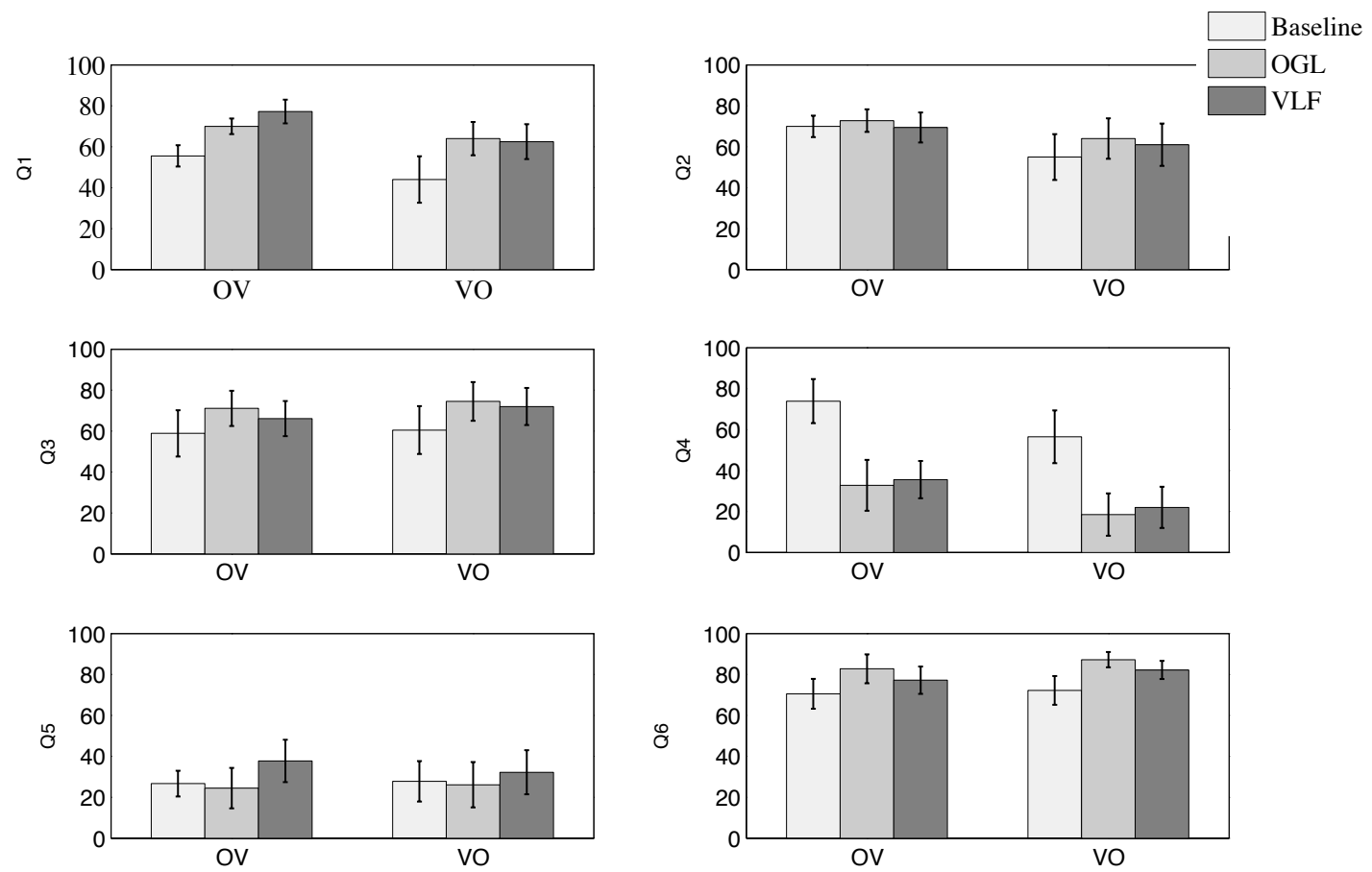

Figure 4 - Bar Charts for the Response Variables - Showing the Mean and Standard Errors for Q1-Q6. OV is the group that experienced the OGL condition first, and VO is the group that experienced the VLF condition first. 


\section{Heart Rate}

We took the middle 2 minutes from the recorded ECG signals of each session and computed the HR and NN50 values. The overall mean $( \pm$ SD) HRs (beats per minute, bpm.) are almost the same between the experimental conditions: $91.8 \pm 16.9 \mathrm{bpm}$ (OGL) and $91.4 \pm 17.0 \mathrm{bpm}$ (VLF). Similarly the mean NN50 counts are almost the same: $10.3 \pm 14.1$ (OGL) and $8.8 \pm 12.5$ (VLF). Thus overall there is no indication of higher stress in one condition compared to another.

However, there is one very interesting result related with HR. We take a conservative measure of the overall level of presence by computing for each individual the number of times that he or she gave a score of at least $70 \%$ on the questions Q1-Q3 and Q6. This transforms the presence scores into a count, and corresponds to what we have used in earlier papers - e.g. [10]. Let's call this the 'presence count' and plot this on the horizontal axis against heart rate on the vertical axis, shown in Figure 5. There is a clear linear positive trend, and the correlation is high $(\mathrm{r}=0.51, \mathrm{P}<0.001, \mathrm{n}=38)$ taking account of missing observations. (There are 20 subjects each of who did the OGL and VLF conditions and thus 40 data points. 2 are lost, 1 each in OGL and VLF, because one participant answered 0 in all questions).

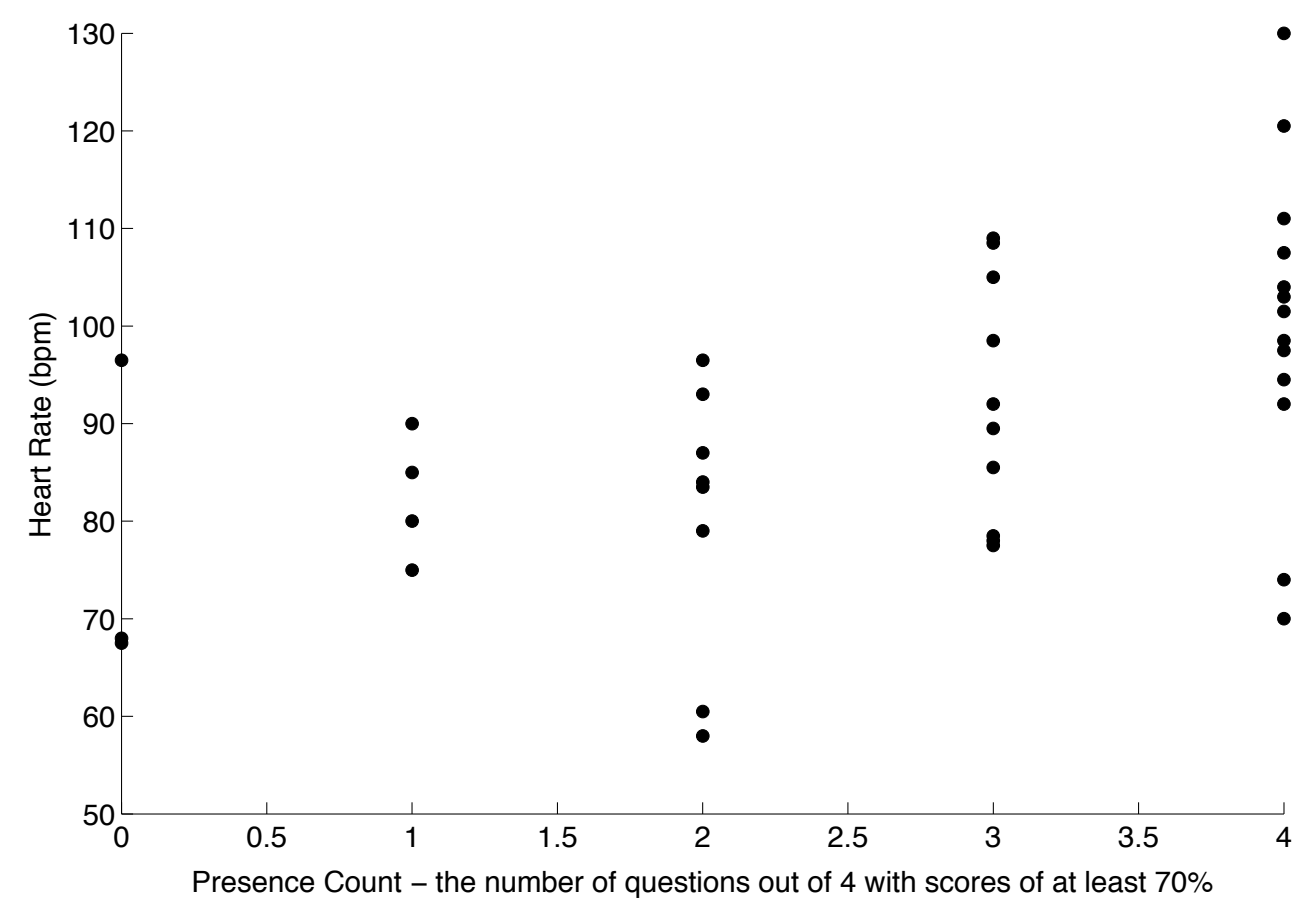

Figure 5 - HR bpm by presence count. 


\subsection{Heart Rate Deceleration}

Focusing in more we can examine the impact of the particular event designed to cause a surprise or shock, which was when the virtual boy floated into the environment. This did produce visible shocks in 10 of the subjects in their first exposure, and some of them actually screamed when they first saw the boy. In order to examine this physiologically, we computed heart rate deceleration (HRD) for the 6s period after the boy entered the environment. The steeper the deceleration the greater indication of negative valence. We compared this with the $6 \mathrm{~s}$ period that started 10s before the boy entered the environment as a control. For this variable we expected an ordering effect, since the second time the full scenario was displayed, there could be a good guess about what would occur.

Table 2

Means and Standard Deviations of the Heart Rate Decelerations for 10s before the Boy Entered and starting from the event itself. The $P$ values are from the sign test $(n=10$ in each cell).

\begin{tabular}{|l|c|c|c|c|c|c|}
\cline { 2 - 7 } \multicolumn{1}{c|}{} & \multicolumn{3}{c|}{ OGL } & \multicolumn{3}{c|}{ VLF } \\
\hline Trial & $\begin{array}{c}10 \mathrm{~s} \\
\text { Before }\end{array}$ & Event & $\mathrm{P}$ & $\begin{array}{c}10 \mathrm{~s} \\
\text { Before }\end{array}$ & Event & $\mathrm{P}$ \\
\hline 2 & $5.4 \pm 5.6$ & $9.3 \pm 20.5$ & 1 & $1.9 \pm 4.3$ & $11.6 \pm 16.4$ & 0.002 \\
\hline 3 & $6.6 \pm 10.1$ & $4.3 \pm 8.1$ & 1 & $6.7 \pm 10.8$ & $5.9 \pm 11.0$ & 1 \\
\hline
\end{tabular}

The HRD values are calculated as the negative of the slope, so greater values indicate greater deceleration. From Table 2 we can see that there is a significant difference between the HRD 10s before the event of the boy entering, and for the event itself - but only for those in the VLF condition. This occurred only when they experienced this event for the first time, that is during their second trial (the first after the Baseline). In this case the mean HDR values are significantly higher for the event than for the $10 \mathrm{~s}$ before the event. Not only is this the case on the average, but it is so for every one of the 10 participants. The fact that there was no significant difference in their second exposure to this event (trial 3) shows that there had been adaptation.

As a further comparison we carried out a similar analysis for the event when the books started to fall. In this case there were no significant differences at all (the lowest $\mathrm{P}$ value amongst those equivalent to Table 2 was 0.11 ). This illustrates that the HRD was able to distinguish between an event that several participants found to be frightening and another event that although it was unusual, had not been designed to have a negative valence. 


\section{Discussion}

Summarizing the immediate results we have found that:

- There are no differences on any presence questionnaire score between OGL and VLF indicating that the level of presence was not influenced by the rendering type.

- There are no differences on overall ECG measures (heart rate and NN50) taken over the middle 2 minutes of the experience, indicating that there was no observable overall physiological difference such as stress between the conditions.

- Heart rate is significantly positively correlated with an overall measure of presence.

- Considering the first exposures only of either the VLF or OGL condition, there is a strong HRD effect with respect to the specific event of virtual boy entering the room in the VLF condition but not in the OGL condition.

If we go back and reconsider the meaning of the experiment that we described in Part 1, it is now clear that it was the dynamic changes to shadows and reflections corresponding to body movements that mattered, and not the quality of the illumination. Hence when the dynamic changes are the same in two conditions but the illumination type varies, as in the new experiment, we do not find this difference in presence.

These findings can be put into the context of a new approach to presence that distinguishes between two different components: Place Illusion (PI) and Plausibility (Psi) $[11,12]$ (see Inset). PI refers to the original idea of presence as the strong illusion of being in the place presented by the virtual reality displays. The new approach suggests PI to be largely a function of the sensorimotor contingencies afforded by the display and tracking systems. The more that people can use their whole bodies to carry out acts of perception in a normal way, the greater the chance for PI to occur. Hence, for example, a wide field-of-view, stereo head-tracked head mounted display is more likely to lead to PI than a narrow field-of-view but otherwise similar system, or through looking around the environment by, say, using a joystick. With respect to the OGL and VLF conditions the affordances for perception were the same - in the Cave with head tracking - and therefore we would not expect to find differences in reported PI as between these two conditions, and this is the case. 
Inset on Place Illusion and Plausibility

The concept of presence has recently been deconstructed into two orthogonal components $[11,12]$ : The first is the pure illusion that participants have that they are located in the place depicted by the virtual environment - this is 'Place Illusion' (PI). The second is the illusion that the events that are apparently happening in that place are really happening. We call this a Plausibility Illusion (Psi). These illusions are produced by our perceptual and cognitive systems, and occur in spite of our high level knowledge that we are, for example, 'really' in a laboratory wearing equipment that generates the sensory data-stream that leads to these illusions. These illusions are automatic, beyond conscious control.

The physical basis of PI is thought to be sensorimotor contingencies that correspond to those of physical reality. Sensorimotor contingencies are the implicit rules that we know concerning how to use our body to perceive the world. For example, we know that to see behind an object we have to move our head to the side to bring parts of the scene that are obscured into view. When someone perceives by carrying out actions that result in changes in (multisensory) images by using their body much as in physical reality, then the simplest hypothesis for the brain to adopt is that what is being perceived is actually there - i.e., that the person is in the place depicted by the virtual environment.

The physical basis of Psi is thought to be the extent to which (a) the application is programmed to produce events that correlate with the behavior of the participant, (b) how much events in the VR refer personally to the participant, and (c) the validity of the scenario with reference to a similar real life situation in which the events depicted might occur.

Why are these illusions necessary for successful VR applications? Imagine you enter into a virtual reality for therapeutic purposes - for example, for overcoming a phobia of speaking in public. The VR puts you in the situation of being in a seminar room standing in front of an audience [A]. If you do not have the illusion that you are there, in that place, no anxiety would be generated. Similarly if you do not have the illusion that there are people there who are responding to your behavior, again it is unlikely that anxiety would be generated. Yet unless some degree of anxiety occurs that is concomitant with what you would experience in a corresponding real place and situation, then it would not be possible to use this application for therapeutic reasons. Some automatically responding part of the brain must have the illusion that you are standing in a room with an audience looking at you, and generate the physiological and emotional responses that go along with this. The fact that you really know that you are not in that place is irrelevant to the success of the application.

To take another example, a social psychologist is carrying out an experiment on how people respond to violent incidents, such as a fight between two people in a bar [B]. Such experiments cannot be carried out in physical reality for ethical and practical 
reasons. However, they can be carried out in virtual reality. For such experiments to be valid the experimental volunteers must have the illusion that they are in the bar where the fight is occurring (PI) and that the fight is really happening (Psi). Without those strong illusions the experimental studies would not be useful, since the appropriate physiological, emotional and behavioral responses would not be forthcoming. Again, we are speaking of automatic responses that occur in spite of your high level cognitive knowledge that in fact you are really in a VR system, and that nothing that is apparently happening is actually happening.

[A] D. P. Pertaub, M. Slater, and C. Barker, "An experiment on public speaking anxiety in response to three different types of virtual audience," PresenceTeleoperators and Virtual Environments, vol. 11, no. 1, pp. 68-78, 2002.

[B] A. Rovira, D. Swapp, B. Spanlang et al., "The use of virtual reality in the study of people's responses to violent incidents," Frontiers in Behavioral Neuroscience, vol. 3:59. doi:10.3389/neuro.08.059.2009, 2009-Dec-23, 2009.

Consider also the strong correlation between heart rate and the overall presence count in the OGL and VLF experimental conditions. Since the overall mean HRs between the two conditions are statistically the same, and since there is no change in heart rate variability as measured by NN50 this correlation result probably reflects the amount of physical activity of participants - the greater the reported presence the greater the amount of activity. Also take into consideration the fact that this relationship between presence and HR can be seen even for the Baseline considered by itself - although not so strongly $(\mathrm{r}=0.42, \mathrm{P}=0.07)$. So the correlation is not caused by the necessity of participants responding to events in the virtual environment, since there are none in the Baseline. Therefore it must be caused by some intrinsic response by participants. This finding does support the idea that PI is associated with natural sensorimotor contingencies, since the only reason for greater body activity is for perception - using the body naturally for perception (e.g., looking and moving around).

Inset on PI and Psi

In the experiment described in [12] there were two groups of participants, one that had been instructed to concentrate only their sensation of being in the virtual place (PI) and the other only on their sensation of the reality of what was happening (Psi). They could attempt to optimize PI or Psi by step-by-step improvements in the quality of a number of properties of the virtual reality system - quality of illumination rendering, field of view, type of display, and self-representation by an avatar. Participants who had been instructed to maximize Psi tended to opt more often and earlier to improve the quality of illumination than those who had been instructed to optimize PI. 
Psi is the illusion that what is happening is real. The experiment reported in Part 1 of this paper can now be interpreted as showing that it was correlations between body movements and corresponding changes in shadows and reflections that was the cause of the greater presence. This is in line with the factors thought to influence Psi as discussed in the Inset - the correlation between participant actions and events in the VR. Additionally we have found that HRD was affected in the VLF condition by the sudden appearance of the ghost-like boy, but that this did not occur in the OGL condition. If participants were tending to treat the situation as real then the sudden appearance of the boy would indeed have been a frightening occurrence and therefore be likely to induce a HRD response. We did find in [12] that there was a greater probability of a Plausibility illusion under the condition of full global illumination with dynamic changes compared with a radiosity-like solution (global illumination with no dynamic changes). Our results from this new experiment support this finding.

Finally, we report that 6 out of the 20 participants did touch their own heads after they saw the book on the top of the mirror avatar head. The mean score for Q7 on feeling that the avatar in the mirror was the self was $58 \pm 27$ (S.D.) with no difference between the OGL and VLF conditions.

\section{Conclusions}

The take-home message of this experiment is that the Place Illusion component of presence is not a function of the quality of illumination rendering. Place Illusion depends on the form through which the environment can be perceived - which is a function of the degree of head and body tracking, and display factors that would support natural perception (field-of-view, resolution, latency, frame-rate, and so on). The affordance of (visual) sensorimotor contingencies that are close to natural, as typically occurs in a Cave system with head-tracking is enough to generate a high level of Place Illusion.

However, if in our application we aim at people responding to the virtual environment realistically, we also have to take into account the Plausibility component of presence. Our results suggest two things. First, where appropriate there should be events that occur in the virtual reality that correlate with the actions of the participants. In the case of our two experiments these events were the dynamically changing shadows and reflections in response to body movements. But other such correlated events are also important - such as virtual characters responding appropriately to the actions of the participant (not considered at all in this paper). Second, do use global illumination - it apparently does help to foster the illusion that the situation depicted is really happening.

So after all we would recommend that real-time global illumination with dynamic changes to reflections and shadows as worth the effort. 


\section{Acknowledgements}

This research was carried out under the UK EPSRC Project 'Presence in a Virtual Light Field' (EP/C511824/1). Mel Slater's contribution was also supported through the European Research Council Project TRAVERSE Contract Number 227985. We thank David Swapp for his invaluable help with the UCL Cave system.

\section{References}

[1] M. Slater, P. Khanna, J. Mortensen et al., "Visual Realism Enhances Realistic Response in an Immersive Virtual Environment," IEEE Computer Graphics and Applications, vol. 29, no. 3, pp. 76-84, 2009.

[2] M. V. Sanchez-Vives, and M. Slater, "From Presence to Consciousness through Virtual Reality," Nature Reviews Neuroscience, vol. 6, no. 4, pp. 332-339, 2005.

[3] P. Zimmons, and A. Panter, "The Influence of Rendering Quality on Presence and Task Performance in a Virtual Environment," IEEE Virtual Reality,. Proceedings., pp. 293-294, 2003.

[4] J. Mortensen, Y. Yu, P. Khanna et al., "Real-Time Global Illumination for Virtual Reality Applications," IEEE Computer Graphics and Applications, vol. 28, no. 6, pp. 56-64, 2008.

[5] R. Ramamoorthi, and P. Hanrahan, "An efficient representation for irradiance environment maps," in ACM SIGGRAPH 2001 Proceedings of the 28th Annual Conference on Computer Graphics and Interactive Techniques, 2001, pp. 497-500.

[6] G. Greger, P. Shirley, P. M. Hubbard et al., "The irradiance volume," IEEE Computer Graphics and Applications, vol. 18, no. 2, pp. 32-43, Mar-Apr, 1998.

[7] Y. Uralsky, "Efficient soft-edged shadows using pixel shader branching," GPU Gems, vol. 2, pp. 269-282, 2005.

[8] M. J. Kilgard, "Improving shadows and reflections via the stencil buffer," NVIDIA White Paper, 2000.

[9] J. Cacioppo, L. Tassinary, and G. Berntson, Handbook of Psychophysiology: Cambridge University Press, 2007.

[10] M. Slater, and A. Steed, "A virtual presence counter," Presence-Teleoperators and Virtual Environments, vol. 9, no. 5, pp. 413-434, 2000.

[11] M. Slater, "Place Illusion and Plausibility Can Lead to Realistic Behaviour in Immersive Virtual Environments," Philos Trans R Soc Lond, vol. 364, no. 1535 pp. 3549-3557, 2009.

[12] M. Slater, B. Spanlang, and D. Corominas, "Simulating Virtual Environments within Virtual Environments as the Basis for a Psychophysics of Presence," ACM Transactions on Graphics (SIGGRAPH) (TOG), vol. 29, no. 3, pp. Paper: 92, 2010. 
Insu Yu is a finishing PhD student at University College London. His interests include real-time global illumination on the GPU, especially based on spherical harmonics. He has an MSc in Vision, Imaging and Virtual Environments (VIVE) from the Department of Computer Science, University College London, and submitted his PhD thesis on the topic of global illumination in June 2011. He can be contacted at i.yu@cs.ucl.ac.uk.

Department of Computer Science

University College London

Gower Street

London WC1E 6BT, UK.

Jesper Mortensen is a Senior Graphics R\&D Engineer at Geomerics Ltd. working on real-time global illumination for the games industry. His research interest is rendering algorithms and particularly global illumination. He has an MSc in Vision, Imaging and Virtual Environments from University College London. He was awarded a PhD on the topic of global illumination at University College London in 2011.

Geomerics Ltd.

City House

126-130 Hills Road

Cambridge

CB2 1RE

Tel: $+44(0) 1223450170$

Fax: +44(0)1223 361362

Email: jesper@geomerics.com

Pankaj Khanna currently works as a consultant in the financial services and maintains links to academia as an Honorary Research Fellow at University College London. He maintains an interest in global illumination, real-time physically-based rendering, and virtual reality. He can be contacted at p.khanna@cs.ucl.ac.uk.

Department of Computer Science

University College London

Gower Street

London WC1E 6BT, UK.

Mel Slater is ICREA Research Professor at the University of Barcelona, Spain, and a Professor of Virtual Environments, University College London, UK. His research interests include virtual reality and its applications in psychology and neuroscience. He has a DSc from University of London and he can be contacted at melslater@ub.edu. 


\section{EVENT Lab \\ Universitat de Barcelona \\ Facultat de Psicologia, \\ Departament de Personalitat, Avaluació i Tractaments Psicològics, \\ Campus de Mundet - Edifici Teatre, \\ Passeig de la Vall d'Hebron 171, \\ 08035 Barcelona \\ Spain}

Tel: +34934039618

Email: melslater@ub.edu 